\title{
Predictors of a positive duplex scan in patients with a clinical presentation compatible with deep vein thrombosis or cellulitis
}

\author{
Curtis E Rabuka MD ${ }^{1}$, Laurent $\mathrm{Y}$ Azoulay $\mathrm{BSc}^{2}$, Susan R Kahn MD MSc ${ }^{2,3}$
}

CE Rabuka, LY Azoulay, SR Kahn. Predictors of a positive duplex scan in patients with a clinical presentation compatible with deep vein thrombosis or cellulitis. Can J Infect Dis 2003;14(4):210-214.

BACKGROUND: Deep vein thrombosis (DVT) and cellulitis are common conditions whose symptoms lead patients to seek medical attention in the emergency department (ED). Distinguishing between these two conditions quickly and accurately is important. OBJECTIVES: To determine the yield of duplex scanning among ED patients whose clinical presentation is compatible with DVT or cellulitis. In addition, to determine whether baseline clinical variables are predictive of the final diagnosis among ED patients with an initial clinical impression of 'DVT versus cellulitis' who underwent duplex scanning.

METHODS: In this historical cohort study, patients with a final diagnosis of DVT (positive duplex) were compared on several baseline variables with patients with a final diagnosis of cellulitis (negative duplex and antibiotics prescribed).

RESULTS: One hundred-nine of 542 ED patients referred for a duplex scan were initially diagnosed as 'DVT versus cellulitis', 17\% of whom had DVT confirmed by a positive duplex scan. Comparing patients with DVT versus those with cellulitis, $0 \%$ versus $15.3 \%$ had rigors $(\mathrm{P}=0.06) ; 0 \%$ versus $8.3 \%$ had distinct margins of erythema $(\mathrm{P}<0.01)$; $5.3 \%$ versus $22.2 \%$ were currently on antibiotics $(P=0.09)$; and $50 \%$ versus $21.3 \%$ had an elevated white blood cell count $(\mathrm{P}=0.04)$.

CONCLUSION: There are differences in a number of baseline characteristics of 'DVT versus cellulitis' patients who went on to have either positive or negative duplex scans, some of which were statistically significant despite the limited sample size. These findings should be confirmed prospectively in a larger study sample since they may have the potential to aid in the clinical differentiation between DVT and cellulitis.

Key words: Cellulitis; Deep vein thrombosis; DVT; Emergency

\section{Prédicteurs d'un écho-Doppler positif chez les patients présentant un tableau clinique com- patible avec celui d'une thrombose veineuse profonde ou d'une cellulite}

CONTEXTE : La thrombose veineuse profonde (TVP) et la cellulite sont deux affections courantes, dont les symptômes amènent les patients à consulter un médecin au service des urgences, et il importe de distinguer vite et bien l'une de l'autre.

OBJECTIFS : L'étude visait d'abord à déterminer le rôle de l'échoDoppler chez les patients à l'urgence dont le tableau clinique était compatible avec celui d'une TVP ou d'une cellulite, puis à vérifier si certaines variables cliniques de base pouvaient servir de prédicteurs de diagnostic final chez les patients qui avaient subi l'examen et qui avaient laissé au départ une impression clinique de TVP ou de cellulite.

MÉTHODE : Dans la présente étude de cohorte historique, nous avons comparé plusieurs variables de base chez les patients ayant reçu un diagnostic final de TVP (écho-Doppler positif) et chez ceux ayant reçu un diagnostic final de cellulite (écho-Doppler négatif et antibiotiques prescrits).

RÉSULTATS : Cent neuf patients sur cinq cent quarante-deux chez qui un diagnostic initial de TVP avait été posé à l'urgence par rapport à celui de cellulite ont subi un écho-Doppler; $17 \%$ d'entre eux ont vu le diagnostic de TVP confirmé par l'examen. L'étude des variable chez les patients souffrant d'une TVP comparativement à ceux souffrant d'une cellulite a donné les résultats suivants : frissons : $0 \%$ contre (c.) 15,3\% $(\mathrm{P}=0,06)$; halo érythémateux bien défini : $0 \%$ c. $8,3 \%(\mathrm{P}<0,01)$; antibiotiques : $5,3 \%$ c. $22,2 \%(\mathrm{P}=0,09)$; leucocytose : $50 \%$ c. $21,3 \%$ $(\mathrm{P}=0,04)$.

CONCLUSION : Des différences ont été relevées quant à certaines caractéristiques de base chez les patients atteints d'une TVP ou d'une cellulite et chez qui l'écho-Doppler s'est avéré positif ou négatif; certaines différences étaient même statistiquement significatives, et ce, malgré la petite taille de l'échantillon. Les résultats obtenus devraient être confirmés dans le cadre d'une étude prospective, plus grande, étant donné que ces caractéristiques peuvent aider à distinguer la TVP de la cellulite en clinique.
$\mathrm{D}$ eep vein thrombosis (DVT) and cellulitis are common conditions whose symptoms lead patients to seek medical attention in the emergency department (ED). Both may have serious consequences. Cellulitis may progress to sepsis, while DVT can lead to pulmonary embolism, which can be fatal, or chronic post-thrombotic syndrome, which can be debilitating. Although the clinical presentations of DVT and cellulitis can be similar, their management is completely different: patients with DVT require urgent anticoagulation whereas those with cellulitis require antibiotics. Hence, distinguishing between

${ }^{1}$ Emergency Medicine Department, ${ }^{2}$ Center for Clinical Epidemiology and Biostatistics, and ${ }^{3}$ Division of Internal Medicine, Sir Mortimer B Davis Jewish General Hospital, McGill University, Montreal, Quebec

Presented at International Conference on Emergency Medicine, Boston, USA, May 2000 and Canadian Association of Emergency Physicians, Saint John, New Brunswick, June 2000

Correspondence: Dr Curtis E Rabuka, Division of Emergency Medicine, Faculty of Medicine and Dentistry, University of Alberta,

1G1.62 Walter Mackenzie Health Sciences Centre, 8440-112 Street, Edmonton, Alberta T6G 2B7. Telephone 780-407-7047,

fax 780-407-3314, e-mail curtisrabuka@shaw.ca

Received for publication January 6, 2003. Accepted May 12, 2003 
TABLE 1

Demographic data

\begin{tabular}{lcc}
\hline & $\begin{array}{c}\text { Negative duplex } \\
\text { (cellulitis) }(\mathbf{n}=\mathbf{7 2})\end{array}$ & $\begin{array}{c}\text { Positive duplex } \\
\text { (DVT) }(\mathbf{n}=19)\end{array}$ \\
\hline Mean age \pm SD & $71.3 \pm 17.4$ & $71.4 \pm 13.7$ \\
Female $\mathrm{n}(\%)$ & $39(54.2 \%)^{\star}$ & $15(78.9 \%)$ \\
\hline
\end{tabular}

${ }^{*} P=0.051$ for difference between groups. DVT Deep vein thrombosis; duplex Duplex ultrasound

TABLE 2

Symptoms and signs, $\mathbf{n}(\%)$

\begin{tabular}{lcc}
\hline & $\begin{array}{c}\text { Negative duplex } \\
\text { (cellulitis) (n=72) }\end{array}$ & $\begin{array}{c}\text { Positive duplex } \\
\text { (DVT) (n=19) }\end{array}$ \\
\hline Chills or rigors & $11(15.3 \%)^{*}$ & $0(0 \%)$ \\
Constitutional symptoms & $13(18.1 \%)$ & $1(5.3 \%)$ \\
Distinct margins of erythema & $6(8.3 \%)^{\dagger}$ & $0(0 \%)$ \\
$\quad$ eg, erysipelas) & $12(17.4 \%)$ & $1(5.9 \%)$ \\
Temperature (at least $\left.38.3^{\circ} \mathrm{C}\right)$ & $(\mathrm{n}=69)$ & $(\mathrm{n}=17)$ \\
Heat rate (at least $100 \mathrm{bpm})$ & $15(22.7 \%)$ & $6(37.5 \%)$ \\
& $(\mathrm{n}=66)$ & $(\mathrm{n}=16)$ \\
Homan's sign & $0(0 \%)^{\ddagger}$ & $1(5.3 \%)$ \\
Varicose veins & $3(4.2 \%)$ & $3(15.8 \%)$ \\
Abscess & $2(2.8 \%)$ & $0(0 \%)$ \\
Red streaking & $3(4.2 \%)$ & $0(0 \%)$ \\
Inguinal lymphadenopathy & $6(8.3 \%)$ & $1(5.3 \%)$ \\
Venous skin changes & $8(11.1 \%)$ & $1(5.3 \%)$ \\
Open wound & $16(22.2 \%)$ & $3(15.8 \%)$ \\
Palpable cord & $2(2.8 \%)$ & $1(5.3 \%)$ \\
Superficial venous dilation & $4(5.6 \%)$ & $0(0 \%)$ \\
\hline
\end{tabular}

${ }^{*} P=0.056, \quad{ }^{\top} P=0.008$, and $\ddagger P=0.014$ for differences between groups. bpm Beats per minute; DVT Deep vein thrombosis

these two diagnoses quickly and accurately is important. Cellulitis is diagnosed on clinical grounds, based on the presence of leg swelling, redness, tenderness, warmth and fever (1). While cellulitis is often easily diagnosed clinically and usually does not require further ED testing, sometimes patients present with a confusing clinical picture compatible with either cellulitis or DVT. Furthermore, in practice a duplex scan may be ordered to rule out DVT even when cellulitis is considered to be the likely diagnosis because the two entities can mimic each other clinically and DVT can only be reliably excluded with objective testing (2).

Despite their similar clinical presentations, studies of diagnostic strategies directly comparing DVT to cellulitis are rare. We performed a literature review from 1976 to 2001 using MEDLINE and found no studies that specifically compared DVT to cellulitis from the perspective of history, physical examination and common initial investigation. One proposed objective diagnostic method for cellulitis, which involves measuring the concentration of protein in edema fluid (3), has not been further validated and is not clinically practical.

If one could identify a group of clinical features that were useful in predicting DVT in patients with clinically suspected cellulitis, it might be possible to achieve quicker, more reliable clinical diagnoses and more rapid access to appropriate therapy. In addition, there would be fewer unnecessary duplex scans in patients who are at low risk for DVT. Conversely, if there were no clinical features that were found to be useful in
TABLE 3

Laboratory data, $\mathbf{n}(\%)$

\begin{tabular}{|c|c|c|}
\hline & $\begin{array}{l}\text { Negative duplex } \\
\text { (cellulitis) }(n=72)\end{array}$ & $\begin{array}{l}\text { Positive duplex } \\
\text { (DVT) }(n=19)\end{array}$ \\
\hline \multirow[t]{2}{*}{ WBC $>11 \times 10^{9} / L$} & $(n=61)$ & $(n=12)$ \\
\hline & $13(21.3 \%)^{*}$ & $6(50 \%)$ \\
\hline Mean & 8.9 & 10.4 \\
\hline \multirow[t]{2}{*}{ Neutrophil $>7.5 \times 10^{9} / \mathrm{L}$} & $(n=61)$ & $(n=12)$ \\
\hline & $21(34.4 \%)$ & $6(50 \%)$ \\
\hline Mean & 6.8 & 7.4 \\
\hline \multirow[t]{2}{*}{ INR $>1.3$} & $(n=22)$ & $(n=9)$ \\
\hline & $11(50 \%)$ & $1(11.1 \%)$ \\
\hline Mean & 2.1 & 1.2 \\
\hline \multirow[t]{2}{*}{$\mathrm{LDH}>220 \mathrm{U} / \mathrm{L}$} & $(n=31)$ & $(n=6)$ \\
\hline & 11 (35.5\%) & $2(33.3 \%)$ \\
\hline Mean & 193.7 & 227.3 \\
\hline \multirow[t]{2}{*}{ CK >195 U/L } & $(n=26)$ & $(n=1)$ \\
\hline & $21(80.8 \%)$ & $0(0 \%)$ \\
\hline Mean & 111.6 & 32 \\
\hline \multirow[t]{2}{*}{ AST >55 U/L } & $(n=31)$ & $(n=6)$ \\
\hline & $2(6.5 \%)$ & $0(0 \%)$ \\
\hline Mean & 23.2 & 30 \\
\hline \multirow[t]{2}{*}{ Platelets $>400 \times 10^{9} / \mathrm{L}$} & $(n=61)$ & $(n=12)$ \\
\hline & $2(3.3 \%)$ & $0(0 \%)$ \\
\hline Mean & 219 & 230.5 \\
\hline
\end{tabular}

${ }^{*} P=0.038$ for difference between groups. AST Aspartate aminotransferase; CK Creatine kinase; DVT Deep vein thrombosis; duplex Duplex ultrasound; INR International normalized ratio; LDH Lactate dehydrogenase; WBC White blood cell count

differentiating DVT from cellulitis, ED physicians could be confident that their use of resources is appropriate when ordering a definitive investigation such as a duplex scan to help differentiate the two conditions.

The objectives of the present study were to determine the yield of duplex scanning among ED patients whose clinical presentations were compatible with DVT or cellulitis and to determine whether baseline clinical variables predicted a positive duplex scan in these patients.

\section{METHODS}

This was a historical cohort study of patients who presented to the ED of Sir Mortimer B Davis Jewish General Hospital between 1995 and 1998 whose clinical presentations were compatible with acute cellulitis of a single lower extremity but who were also referred for a duplex scan, which is the principal diagnostic test used at this institution to diagnose DVT. Study patients had to have had a duplex scan ordered from the ED. Patients were eligible for inclusion if they were referred from the ED for a duplex scan and the treating physician documented a clinical suspicion of cellulitis by writing the term cellulitis, soft tissue infection, skin infection, erysipelas or lymphangitis in the differential diagnosis. Alternatively, patient eligibility was also determined by a clinical description in the original diagnosis that included all four elements of the currently accepted criteria for the clinical diagnosis of cellulitis, namely erythema or redness, pain or tenderness, swelling or edema and a hot or warm area $(1,4,5)$. Patients who did not have clear documentation of cellulitis findings were eligible if they were 
assigned a final diagnosis of cellulitis and were treated with antibiotics after the duplex scan was found to be negative. Patients who had bilateral leg symptoms were excluded because neither cellulitis nor DVT typically present with bilateral symptoms.

The hospital's research ethics committee approved the study protocol before its initiation. A list of all patients who had a duplex ultrasound ordered from the ED between March, 1995 and March, 1998 was obtained from the vascular laboratory. The hospital charts of these patients were screened for the above inclusion criteria. Each chart meeting the inclusion criteria was reviewed in detail by one of the investigators using a standardized data extraction form. Only those baseline data on demographic factors (Table 1), history, physical examination (Table 2) and laboratory assessment (Table 3) that were documented before duplex scanning was performed were extracted. The vascular lab technician performing the duplex scan did not have access to the patients' clinical data.

The baseline data from patients with a positive duplex study (confirmed DVT) were examined and compared to patients with negative duplex studies who subsequently were prescribed antibiotics (presumed cellulitis). The final diagnoses in patients with negative duplex scans who were not prescribed antibiotics were also recorded. For continuous variables, means were calculated and compared between groups using Student's t-test. For categorical values the proportions in each group were calculated and compared using the $\chi^{2}$ test. A 2 -tailed $\mathrm{P}$ value of 0.05 or less was considered significant.

\section{RESULTS}

Of 542 consecutive ED patients who were referred to the vascular laboratory for a duplex scan, 109 (20.1\%) met the study entry criteria (ie, were given an initial clinical diagnosis compatible with cellulitis). Of these, 19 (17.4\%) had a positive duplex scan (ie, final diagnosis was DVT), two (1.8\%) of whom were also treated with antibiotics (ie, final diagnosis was both DVT and cellulitis). Of the patients with negative duplex scans $(n=90), 72(80 \%)$ were subsequently prescribed antibiotics (ie, final diagnosis was cellulitis), while 18 (20\%) were not prescribed antibiotics and were assigned a final diagnosis of Baker's cyst, superficial phlebitis or musculoskeletal injury.

Comparison of the baseline variables in patients with a final diagnosis of DVT versus cellulitis is presented below.

\section{Demographic variables}

The mean age of patients in the cellulitis and DVT groups did not differ, however, there was a trend toward a greater proportion of females in the DVT group compared with the cellulitis group (Table 1).

\section{Symptoms and signs}

A history of chills and/or rigors was obtained in 15.3\% of cellulitis patients versus $0 \%$ of DVT patients $(\mathrm{P}=0.056)$. In addition, constitutional symptoms such as malaise, anorexia, nausea or vomiting were found in $18.1 \%$ of cellulitis patients versus $5.3 \%$ of DVT patients, but this difference did not reach statistical significance. With regard to physical signs, distinct margins of erythema were found in $8.3 \%$ of cellulitis patients versus $0 \%$ of DVT patients ( $\mathrm{P}=0.008)$. Homan's sign was noted in one patient with DVT and in none of the patients with cellulitis. Varicose veins were noted in $15.8 \%$ of DVT patients versus $4.2 \%$ of cellulitis patients, although this difference did not reach statistical significance. There were no significant differences between patient groups for such physical signs as tachycardia, fever, abscess, red streaking, inguinal lymphadenopathy, chronic venous skin changes, open wound, palpable cord, or superficial venous dilation (Table 2).

\section{Comorbid conditions and medications}

Although not reaching statistical significance, current antibiotic use and diabetes were more common in patients with cellulitis than in those with DVT (22\% versus $5.3 \%$, and $29 \%$ versus $16 \%$, respectively), whereas peripheral vascular disease and recent surgery were more common in patients with DVT than in those with cellulitis (32\% versus $15 \%$, and $26 \%$ versus $1 \%$, respectively). For variables such as current use of anticoagulants, estrogen-containing medications or corticosteroids, past history of cellulitis, DVT or malignancy, and recent trauma, there were no differences between the patient groups (data not shown).

\section{Laboratory data}

Leukocytosis (defined in Table 3 as greater than $11 \times 10^{9} / \mathrm{L}$ ) was detected in $50 \%$ of DVT patients versus $21.3 \%$ of cellulitis patients $(\mathrm{P}=0.038)$. There were no differences between patient groups with respect to mean platelet count, mean levels of lactate dehydrogenase or aspartate aminotransferase, or the proportions of patients with abnormal cut-off values for these tests. Although mean creatine kinase $(\mathrm{CK})$ was higher in the cellulitis group, only one patient in the DVT group had their CK levels measured (Table 3).

\section{DISCUSSION}

We found that over a three-year period, 20\% of patients presenting to the ED who had a duplex scan ordered were also suspected to have cellulitis, affirming that differentiating cellulitis from DVT is a common clinical dilemma. Among these, $17.4 \%$ had DVT confirmed by a duplex scan, while only $1.8 \%$ had both DVT and cellulitis.

Among study patients, there were differences in the clinical features of patients who had positive duplex scans (ie, DVT) and those who had negative duplex scans (ie, cellulitis). Specifically, we found that patients with a diagnosis of cellulitis were more likely to have constitutional symptoms, rigors, a past history of diabetes and/or distinct margins of erythema (we examined whether distinct margins of erythema might distinguish skin infection from DVT, since this finding is not a typical feature of DVT but may be associated with erysipelastype skin infections). Since cellulitis is a skin infection, these findings are not unexpected, but rather confirm that it is important to elicit these elements of the history and physical examination in patients suspected of having DVT or cellulitis. Diabetics are at increased risks for skin infections for a number of reasons, hence they are, on average, more prone to cellulitis. Conversely, diabetes is not a known risk factor for DVT. More of the patients with a final diagnosis of cellulitis were on antibiotics at the time of presentation than were those with a final diagnosis of DVT. This is likely explained by a previous diagnosis of cellulitis with a prescription of antibiotic therapy and subsequent treatment failure, leading to an ED visit for further evaluation.

Patients with a final diagnosis of DVT were more likely to have had recent surgery, a history of varicose veins and/or peripheral vascular disease, all of which are considered risk factors for DVT. However, it was surprising and noteworthy 
that more than twice the proportion $(50 \%$ versus $21.3 \%$ ) of patients with a final diagnosis of DVT, compared with cellulitis, had an elevated WBC count. None of the other laboratory data examined showed a difference between the two groups, although there was a suggestion that CK levels may be higher in patients with cellulitis, which merits further study.

Previous studies have proposed methods to objectively diagnose cellulitis, including soft tissue imaging with magnetic resonance imaging $(6,7)$ or nuclear isotope scan $(8)$. These are not commonly used in the clinical setting. Aspiration and culture of fluid from the leading edge of the affected area has proven to be very limited as a diagnostic method (1). Most often, cellulitis is diagnosed on clinical grounds, in some cases only after DVT has been objectively ruled out. Ginsberg (9) reviewed 101 cases of cellulitis to better define the presentation, diagnosis, treatment and prognosis of cellulitis. While describing various clinical characteristics of his patients with cellulitis, his study did not specifically compare cellulitis with DVT. Glover and Berdick (10) found that among 245 patients with cellulitis, none had a "clinically significant" DVT on a duplex scan. However, their study population was limited to patients with "clinically evident cellulitis", which may be different from the population in the present study. Mahe et al (11) found that six of 40 patients with a diagnosis of erysipelas had a clinically significant DVT on duplex scan, similar to our findings.

Contrast venography is the accepted 'gold standard' for the objective diagnosis of DVT. However, venous duplex ultrasonography has increasingly become the first-line diagnostic test for DVT, since, unlike contrast venography, it is non-invasive. Previous studies have noted the sensitivity and specificity of duplex scans to be up to $97 \%$ (12). Miller et al (13) found that duplex scans, when compared with contrast venography, were highly accurate in diagnosing acute DVT of the lower extremities, avoiding contrast venography in over $90 \%$ of the cases. Duplex scanning is now the first choice investigation of many ED physicians for the diagnosis of DVT $(4,10,13)$ and as its accuracy has improved, so has its usage. Glover and Bendick (10) evaluated results of duplex scans to determine the appropriate indications for this test. They concluded that there was overuse of duplex scanning and suggested that the history and physical examination should be important indicators of the presence or absence of lower extremity DVT. While they provide some clinical guidelines that help to determine if a patient is a candidate for duplex scanning, they do not specifically identify criteria to differentiate between cellulitis and DVT.

To make the diagnosis of DVT we used only duplex ultrasonography. D-dimer testing has gained increasing utility and acceptance as an ancillary diagnostic tool for thromboembolic disease $(14,15)$. Rapid D-dimer testing, however, has only become widely available during the past few years and was not used at our institution at the time of this study, hence, we could not evaluate its use in the diagnostic process.

Our study has several limitations. Since this was a retrospective study, the data we collected may have been incomplete or imprecise. It is possible that the actual study population was different from the target study population (ie, patients in whom the initial clinical impression was restricted to DVT versus cellulitis). However, since the charts of all of the ED patients sent for duplex scans were reviewed for possible entry into the study, we were unlikely to miss patients in whom DVT was suspected. On the other hand, if, because of poor documentation, it was not apparent that cellulitis was in the differential diagnosis, such patients could have been inadvertently excluded from the study (ie, we may have selected for those patients who had more severe symptoms or signs of cellulitis, which as a result were better documented). We attempted to avoid overselection of severe cases by allowing for many different terms in the chart that could indicate cellulitis. Because the 'gold standard' for the diagnosis of cellulitis is a clinical impression based on cardinal signs and symptoms, we felt this was the most appropriate way to select patients for entry into the study. All data on potential predictor variables were obtained from the hospital chart, hence, some data were missing. However, this was expected to occur in a non-differential fashion across outcome groups. Also, much of the data we examined, such as vital signs, results of lab tests, and information on previous hospitalizations and medical conditions, were universally available in the hospital records.

Measurement bias could have occurred if we extracted data that were recorded on the ED chart after the ED physician knew the duplex result, however, we did not use data on predictor variables recorded after the results of the duplex scan were known.

Another source of possible measurement bias was the data collection process, since one investigator collected all of the data. As it was not possible to blind the investigator to the results of the duplex scans, there existed the possibility of the investigator reporting data differently in different groups. To reduce this bias, we developed very specific criteria for data collection using a standardized case report form.

Regarding generalizability of the study findings: while we only accrued patients from one site, our hospital is a university teaching hospital that also serves as a community hospital to a local catchment area, and the patients it services are highly diverse.

This paper was designed to examine patients with acute symptoms. Despite our best attempts, the retrospective design of the study did not allow us to ensure that all patients with chronic problems were excluded. However, since these were acute care visits to an emergency department it was assumed that the patients were seeking attention for an acute problem when not otherwise stated on the chart.

As is often the case in the ED, patients are initially assigned a diagnosis and treatment, and follow-up is conducted by a different care provider. It is therefore possible that after continued follow-up the diagnosis for some patients may have proven to be different. This limitation was not addressed in the present retrospective analysis but could be dealt with in a prospective follow-up study.

\section{CONCLUSION}

We found that $17.4 \%$ (19 of 109) of patients with suspected cellulitis referred for a duplex scan had a positive scan result. We found differences in a number of the baseline characteristics of 'DVT versus cellulitis' patients who had positive versus negative duplex scans, some of which were statistically significant despite the limited sample size. These findings, if confirmed prospectively in a larger study, may aid clinicians in the diagnosis of cellulitis in patients eliciting an initial clinical impression of cellulitis versus DVT.

ACKNOWLEDGEMENT: Dr Kahn is a Clinical Research Scholar supported by the Fonds de la Recherche en Santé du Québec. 


\section{REFERENCES}

1. Swartz M. Chapter 78 - Cellulitis and subcutaneous tissue infections. In: Mandell GL, Bennett JE, Dolin R, eds. Douglas and Bennett's Principles and Practice of Infectious Diseases, 5th edn. New York: Churchill Livingstone, 2000:1037-50.

2. Heijboer H, Cogo A, Buller HR, Prandoni P, ten Cate JW. Detection of deep vein thrombosis with impedance plethysmography and realtime compression ultrasonography in hospitalized patients.

Arch Intern Med 1992;152:1901-3.

3. Berlyne GM, Kwan T, Li J, Caniso C. Edema protein concentrations for differentiation of cellulitis and deep vein thrombosis. Lancet 1989;ii:728-9.

4. Young JR. The swollen leg. Cardiol Clin 1991;9:443-56.

5. Merli GJ, Spandorfer J. The outpatient with unilateral leg swelling. Med Clin North Am 1995;79:435-47.

6. Saiag P, Le Breton C, Pavlovic M, Fouchard N, Delzant G, Bigot JM. Magnetic resonance imaging in adults presenting with severe acute infectious cellulitis. Arch Dermatol 1994;130:1150-8.

7. Rahmouni A, Chosidow, O, Mathieu, D, et al. MR imaging in acute infectious cellulitis. Radiology 1994;192:493-6.

8. Becker W, Bair J, Behr T, et al. Detection of soft-tissue infections and osteomyelitis using a technetium-99m-labelled anti-granulocyte monoclonal antibody fragment. J Nucl Med 1994;35:1436-43.

9. Ginsberg, MB. Cellulitis: Analysis of 101 cases and review of the literature. South Med J 1981;74:530-3.

10. Glover JL, Bendick PJ. Appropriate indications for venous duplex ultrasonographic examinations. Surgery 1996;120:725-31.

11. Mahe A, Destelle JM, Bruet A, et al. [Deep venous thrombosis in patients with erysipelas of the leg]. Presse Med 1992;21:1022-4.

12. Lensing AWA, Pandoni P, Brandjes DPM, et al. Detection of deep vein thrombosis by real-time B-mode ultrasonography. N Engl J Med 1989;320:342-5.

13. Miller N, Satin R, Tousignant L, Sheiner NM. A prospective study comparing duplex scan and venography for diagnosis of lowerextremity deep vein thrombosis. Cardiovasc Surg 1996;4:505-8.

14. Wells PS, Brill-Edwards P, Stevens P, et al. A novel and rapid wholeblood assay for D-dimer in patients with clinically suspected deep vein thrombosis. Circulation 1995;91:2184-7.

15. Wells PS, Anderson DR, Bormanis J, et al. SimpliRED D-dimer can reduce the diagnostic tests in suspected deep vein thrombosis. Lancet 1998;351:1405-6. 


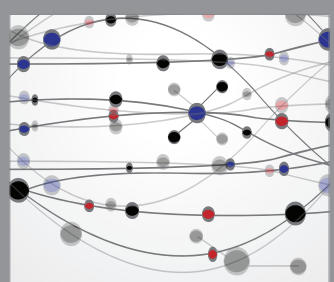

The Scientific World Journal
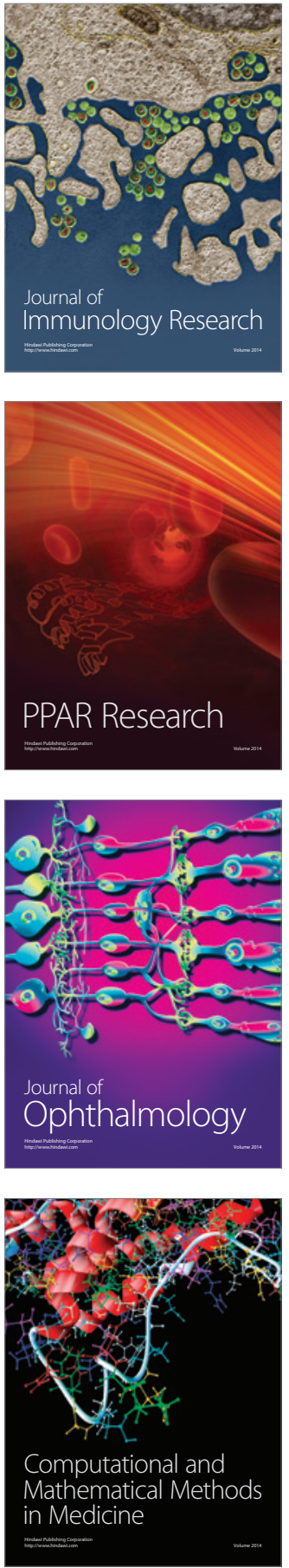

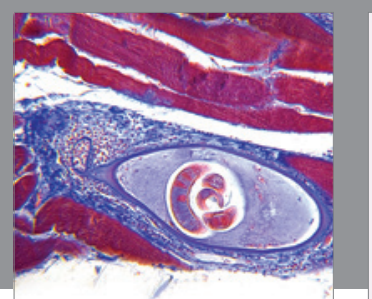

Gastroenterology Research and Practice

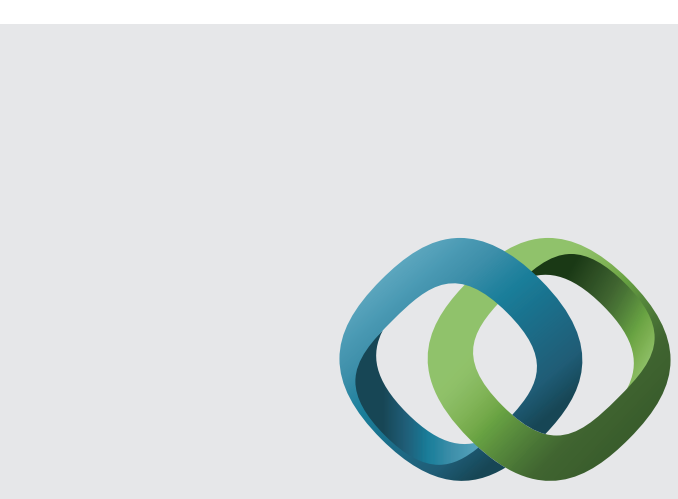

\section{Hindawi}

Submit your manuscripts at

http://www.hindawi.com
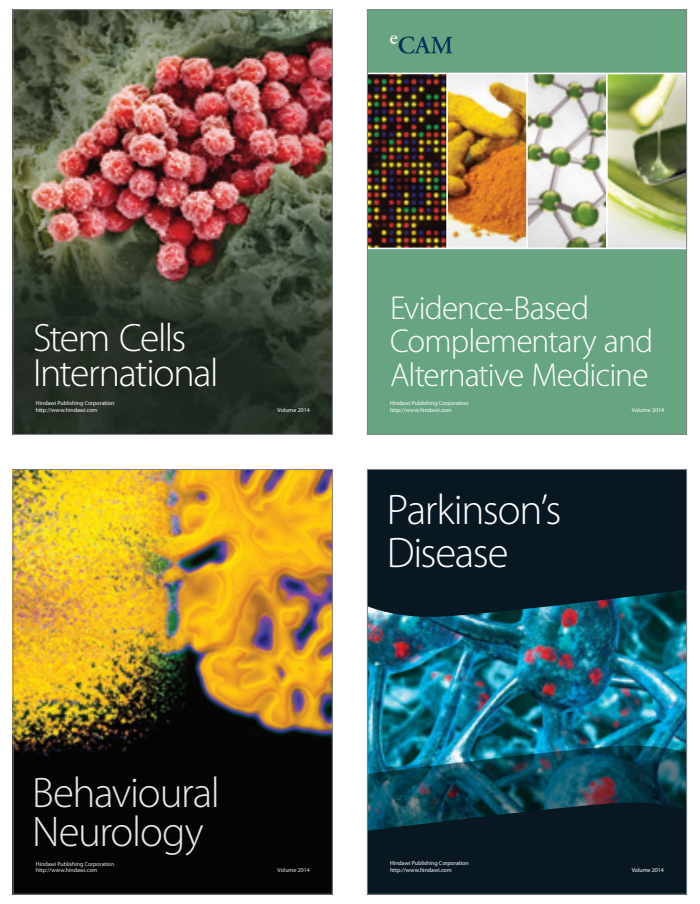
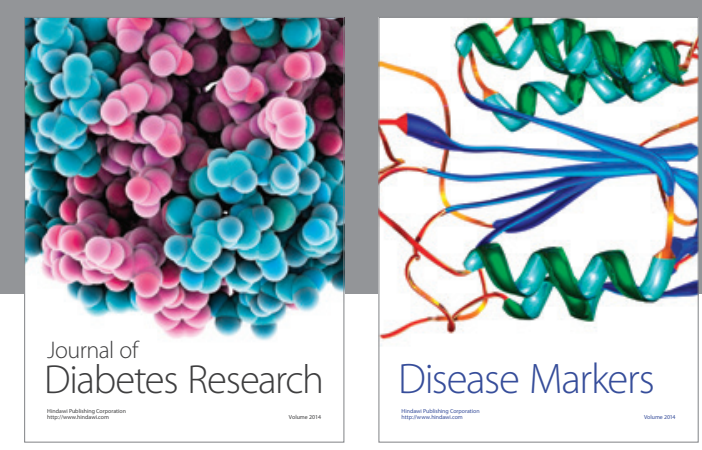

Disease Markers
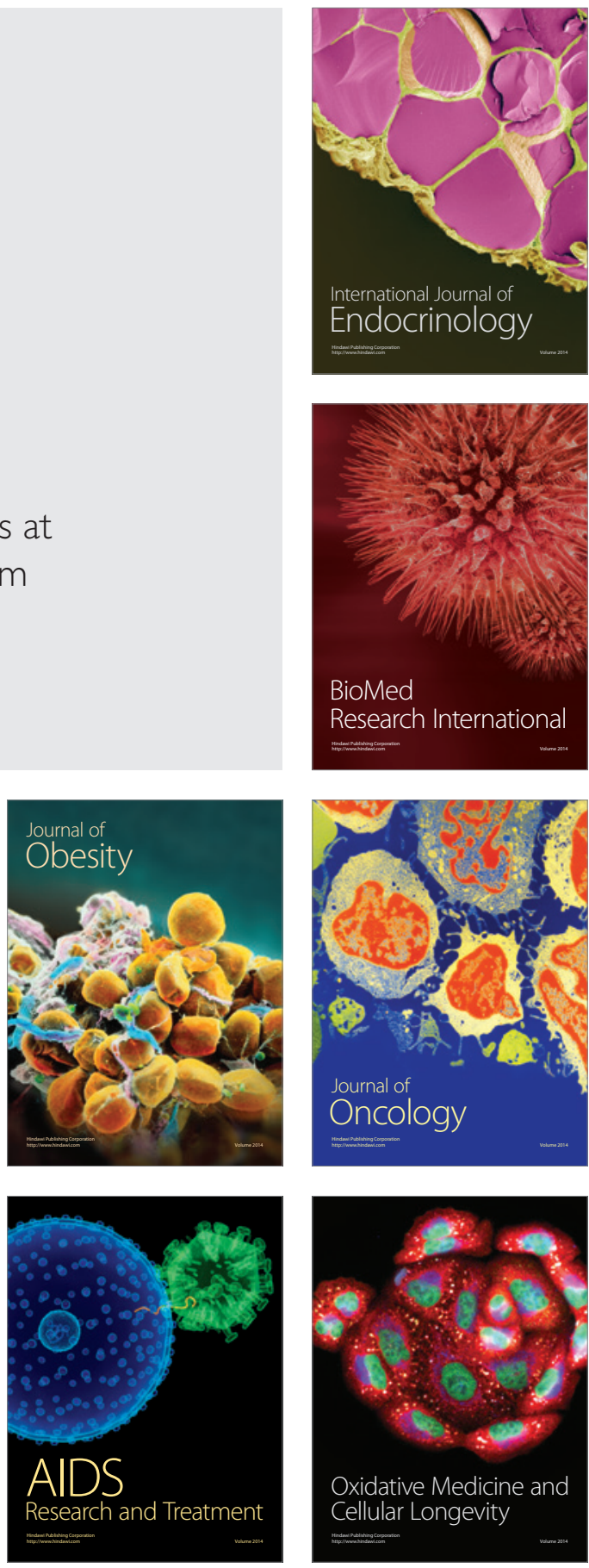